\title{
Corrections of two-photon interactions in the fine and hyperfine structure of the P-energy levels of muonic hydrogen
}

\author{
A. E. Dorokhov* \\ Joint Institute of Nuclear Research, BLTP, \\ 141980, Moscow region, Dubna, Russia \\ N. I. Kochelev \\ Institute of Modern Physics of Chinese Academy of Sciences, 730000, Lanzhou, China and \\ Joint Institute of Nuclear Research, BLTP, \\ 141980, Moscow region, Dubna, Russia \\ A. P. Martynenko ${ }^{\dagger}$ and F. A. Martynenko \\ Samara University, 443086, Samara, Russia \\ A. E. Radzhabov ${ }^{\ddagger}$ \\ Institute of Modern Physics, Chinese Academy of Sciences, Lanzhou 730000, China and \\ Matrosov Institute for System Dynamics and Control Theory SB RAS, 664033, Irkutsk, Russia \\ In the framework of the quasipotential method in quantum electrodynamics we \\ calculate corrections to the nuclear structure proportional to $r_{N}^{2}$ from two-photon \\ exchange amplitudes in the fine and hyperfine structure of P-states in muonic hydro- \\ gen, as well as the photon-photon interaction amplitudes, leading to the exchange \\ of the axial vector meson. In constructing the quasipotential of the muon-nucleus \\ interaction, we use the method of projection operators on states of two particles with \\ a definite spin and total angular momentum. Analytical calculation of the matrix \\ elements is performed and contributions to the fine and hyperfine structure of the \\ $2 P_{1 / 2}$ and $2 P_{3 / 2}$ levels are obtained. \\ PACS numbers: 31.30.Jv, 12.20.Ds, 32.10.Fn
}

\section{INTRODUCTION}

The recent studies of the Lamb shift and hyperfine structure of the spectrum of muonic hydrogen, deuterium and helium have opened a new stage in precise investigation of the energy spectra of simplest atoms. Experiments performed by the CREMA collaboration (Charge Radius Experiments with Muonic Atoms) [1-4] made it possible to carry out ad-

\footnotetext{
*E-mail: dorokhov@theor.jinr.ru

$\dagger$ E-mail: a.p.martynenko@samsu.ru

$\ddagger$ E-mail: aradzh@icc.ru
} 
ditional testing of the Standard Model, to obtain more accurate values of a number of fundamental parameters, and to raise the question of the presence of additional exotic interactions between particles in the bound state. Inclusion of other experimental groups in this area of research (see [5 7]) will allow not only to check the experimental results of the CREMA collaboration, but will also lead, as planned, to further increasing the accuracy of measurements of different intervals of fine and hyperfine structure. The measurements already performed by the CREMA collaboration show that there is a significant difference between the values of such a fundamental parameter as the proton (deuteron) charge radius obtained in the study of the electron and muonic atoms [3, 4, 8- 10]. It should be noted that recent study [11] of the $(2 S-4 P)$ transition in the electronic hydrogen atom yielded a new value of the proton charge radius, $r_{p}=0.8327(87) \mathrm{fm}$, which is quite consistent with the CREMA data. But new measurement of the transition frequency $(1 S-3 S)$ in hydrogen, carried out in Ref. [12], gave the value of the proton charge radius $r_{p}=0.877(13) \mathrm{fm}$, which completely agrees with the CODATA value. As was often the case in long history of precision studies of the energy spectra of simplest atoms in quantum field theory, one of the ways to overcome the crisis situation involves a deeper theoretical analysis, testing previously calculated contributions that can play a large role in the case of muonic atoms. On this way, the problem of a more accurate theoretical construction of the particle interaction operator in quantum electrodynamics, the calculation of new corrections in the energy spectra of muonic atoms acquire a particularly important value [13 18].

In this paper, we investigate contributions of some two-photon interactions in muonic hydrogen. The first part of two-photon exchange interactions is the corrections to the finite size of the nucleus in the fine and hyperfine structure of the P-energy levels. If in the case of S-energy levels these two-photon amplitudes have been studied in sufficient detail by different authors [19 26], then for P-energy levels such detailed calculations have not actually been carried out. Since the overlapping region of the muon wave function and the nucleus for P-states is significant, such contributions may be essential for a more accurate comparison of theory and experiment. This paper continues our series of studies of corrections to the structure of the nucleus without recoil [13 15] for the case of P-energy levels. It is well known that corrections to the structure and polarizability of the nucleus play an important role in the comparison of theory and experiment, since the experimental accuracy of measuring the atomic energy levels is constantly increasing. Periodically arising discrepancies between theory and experiment are usually attributed precisely to corrections for the structure of the nucleus, since there are always significant errors in their calculation. The second part of the photon-photon interactions under consideration is related with the appearance of an effective exchange of the axial vector meson between the muon and proton which leads to shifts of P-levels of muonic hydrogen. In the case of S-states interactions of this type are significant [16, 17]. The purpose of this part of the work is to understand how important the meson exchange processes can be to clarify the position of the P-energy levels.

\section{GENERAL FORMALISM}

To investigate the fine and hyperfine structure of the energy spectrum of muonic hydrogen, we use a quasipotential method in quantum electrodynamics in which the bound state of a muon and a proton is described in the leading order in the fine-structure constant by the Schrödinger equation with the Coulomb potential [27 29]. The first part of important corrections in the energy spectrum of the S- and P-states is determined by the Breit Hamil- 
tonian [27, 28, 30] (hereinafter abbreviated "fs" and "hfs" for the contribution to the energy spectrum of fine structure and hyperfine structure):

$$
\begin{gathered}
H_{B}=H_{0}+\Delta V_{B}^{f s}+\Delta V_{B}^{h f s}, \quad H_{0}=\frac{\mathbf{p}^{2}}{2 \mu}-\frac{Z \alpha}{r} \\
\Delta V_{B}^{f s}=-\frac{\mathbf{p}^{4}}{8 m_{1}^{3}}-\frac{\mathbf{p}^{4}}{8 m_{2}^{3}}+\frac{\pi Z \alpha}{2}\left(\frac{1}{m_{1}^{2}}+\frac{1}{m_{2}^{2}}\right) \delta(\mathbf{r})-\frac{Z \alpha}{2 m_{1} m_{2} r}\left(\mathbf{p}^{2}+\frac{\mathbf{r}(\mathbf{r p}) \mathbf{p}}{r^{2}}\right)+ \\
+\frac{Z \alpha}{2 m_{1}^{2} r^{3}}\left[1+\frac{2 m_{1}}{m_{2}}+2 a_{\mu}\left(1+\frac{m_{1}}{m_{2}}\right)\right]\left(\mathbf{L s}_{1}\right), \\
\Delta V_{B}^{h f s}=\frac{8 \pi \alpha \mu_{p}}{3 m_{1} m_{2}}\left(\mathbf{s}_{1} \mathbf{s}_{2}\right) \delta(\mathbf{r})-\frac{\alpha \mu_{p}\left(1+a_{\mu}\right)}{m_{1} m_{2} r^{3}}\left[\left(\mathbf{s}_{1} \mathbf{s}_{2}\right)-3\left(\mathbf{s}_{1} \mathbf{n}\right)\left(\mathbf{s}_{2} \mathbf{n}\right)\right]+ \\
\frac{\alpha \mu_{p}}{m_{1} m_{2} r^{3}}\left[1+\frac{m_{1}}{m_{2}}-\frac{m_{1}}{2 m_{2} \mu_{p}}\right]\left(\mathbf{L s}_{2}\right)
\end{gathered}
$$

where $m_{1}, m_{2}$ are the masses of a muon and proton correspondingly, $\mu_{p}$ is the proton magnetic moment in nuclear magnetons, $\mathbf{s}_{1}$ and $\mathbf{s}_{2}$ are the muon and proton spins. The contribution of interactions (11)-(3) to the energy spectrum of different muonic atoms was well investigated [19, 21, 31 36]. The interaction operator (3) gives the basic contribution of order $\alpha^{4}$ to hyperfine structure of muonic atom (the Fermi energy). The precise calculation of hyperfine structure, which is necessary for a comparison with experimental data, requires the consideration of various corrections.

An infinite series of perturbation theory for the particle interaction operator contains contributions of different orders in $\alpha$ (the fine structure constant). One such contribution due to the two-photon exchange interaction is investigated in this paper. The amplitudes of this interaction are shown in Fig. 1.

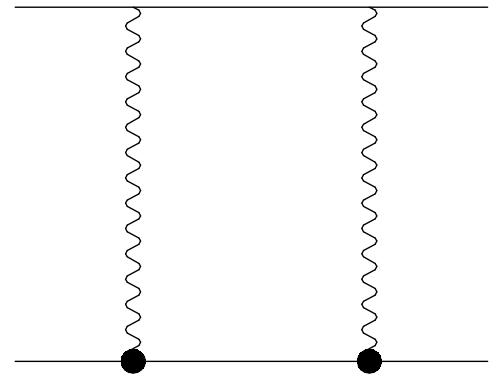

a

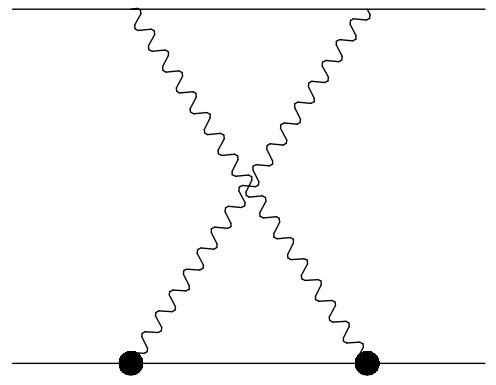

b

FIG. 1: Direct and crossed amplitudes of the two-photon exchange interaction. Thick dot in the diagram indicates the vertex operator of nucleus.

The two-photon exchange interaction amplitudes determine an important next to leading order contribution to the energy spectrum of muonic atom. The corrections of this interaction were studied in detail in the case of S-states both in the Lamb shift and in the hyperfine structure of the spectrum. The vertex of the electromagnetic interaction of a proton (nucleus) is determined by two electromagnetic form factors, information about which was obtained in the study of the scattering of leptons by protons (nuclei). Usually when 
studying this scattering the one-photon approximation is considered, in which the proton (nucleus) is in initial and final states on the mass shell. In the amplitudes in Figure1 in each of the two vertices of the electromagnetic interaction there is a line of the nucleus, which denotes the virtual state of the proton (nucleus). Therefore, the use of free proton form factors at these vertices always caused criticism and led to theoretical errors, which in the case of muonic atoms are much larger than for electron atoms. At present, different methods are used to overcome these difficulties: the dispersion approach, effective field theories [22 26]. For P-states, the calculation of energy corrections was discussed to a much lesser degree than for the S-levels, so one of the goals of this paper is to fill this gap.

For the construction of the particle interaction operator we use the tensor method [13, 14] based on the tensor representation of wave functions and projection operators for P-states of muonic hydrogen. It is convenient to work further in the momentum representation, in which the 2P-state wave function of the muonic hydrogen atom has the form:

$$
\psi_{2 P}(\mathbf{p})=\left(\varepsilon \cdot n_{p}\right) R_{21}(p)
$$

where $\varepsilon_{\delta}(L)$ is the polarisation vector of orbital motion with the normalization condition $\varepsilon^{2}=3 / 4 \pi, n_{p}=(0, \mathbf{p} / p), R_{21}(p)$ is the radial wave function in momentum representation. Corrections to the energy levels are then determined in integral form:

$$
\Delta E\left(2^{2 F+1} P_{J}\right)=\int\left(\varepsilon^{*} \cdot n_{q}\right) R_{21}(q) \frac{d \mathbf{q}}{(2 \pi)^{3 / 2}} \int\left(\varepsilon \cdot n_{p}\right) R_{21}(p) \frac{d \mathbf{p}}{(2 \pi)^{3 / 2}} \Delta V(\mathbf{p}, \mathbf{q}) .
$$

In the leading order the potential of fine or hyperfine structure $\Delta V(\mathbf{p}, \mathbf{q})$ is constructed by means of the amplitude of one-photon interaction $T_{1 \gamma}$ and is determined by formula (3). Starting with the two-photon interaction amplitudes, it is convenient to use the tensor method [13, 14] to construct the interaction potential. It consists in the fact that in the interaction amplitude of particles we consistently perform the projection on the muon states with the total angular momentum $\mathbf{J}$ equal to $1 / 2$ or $3 / 2$, and then the projection of the muon-proton system to states with the total angular momentum $\mathbf{F}=\mathbf{J}+\mathbf{s}_{2}$. As a result of these actions, it appears that the contribution to the particle interaction operator for a given state is determined by the trace of the product of certain $\gamma$ factors, whose calculation and simplification can be performed for complex amplitudes using the Form [37] package. Let us clarify the realization of this calculation method using the amplitude example in Fig. 1. Direct and crossed amplitudes have the following form:

$$
\begin{gathered}
i \mathcal{M}_{1}=\left(Z e^{2}\right)^{2} \int \frac{d^{4} k}{(2 \pi)^{4}}\left[\bar{u}_{1}\left(q_{1}\right) \gamma_{\mu} \frac{\left(\hat{p}_{1}+\hat{k}+m_{1}\right)}{\left(p_{1}+k\right)^{2}-m_{1}^{2}} \gamma_{\nu} u\left(p_{1}\right)\right] \frac{g_{\mu \lambda}}{(k-p)^{2}} \frac{g_{\nu \sigma}}{(k-q)^{2}} \times \\
{\left[\bar{v}\left(p_{2}\right) \Gamma_{\sigma} \frac{\left(\hat{k}-\hat{p}_{2}+m_{2}\right)}{\left(k-p_{2}\right)^{2}-m_{2}^{2}} \Gamma_{\lambda} v\left(q_{2}\right)\right],} \\
i \mathcal{M}_{2}=\left(Z e^{2}\right)^{2} \int \frac{d^{4} k}{(2 \pi)^{4}}\left[\bar{u}_{1}\left(q_{1}\right) \gamma_{\mu} \frac{\left(\hat{p}_{1}+\hat{k}+m_{1}\right)}{\left(p_{1}+k\right)^{2}-m_{1}^{2}} \gamma_{\nu} u\left(p_{1}\right)\right] \frac{g_{\mu \lambda}}{(k-p)^{2}} \frac{g_{\nu \sigma}}{(k-q)^{2}} \times \\
{\left[\bar{v}\left(p_{2}\right) \Gamma_{\lambda} \frac{\left(-\hat{k}-\hat{p}_{2}+m_{2}\right)}{\left(k+p_{2}\right)^{2}-m_{2}^{2}} \Gamma_{\sigma} v\left(q_{2}\right)\right],}
\end{gathered}
$$


where $p_{1,2}=m_{1,2} v \pm p$ are four-momenta of a muon and proton in initial state, $q_{1,2}=m_{1,2} v \pm q$ are four-momenta of a muon and proton in final state, $v_{\mu}=(1,0,0,0)$. The proton vertex operators in direct and crossed amplitudes are equal

$\Gamma_{\sigma}=\gamma_{\sigma} F_{1}\left((k-p)^{2}\right)+\sigma_{\sigma \rho} \frac{(k-p)_{\rho}}{2 m_{2}} F_{2}\left((k-p)^{2}\right), \quad \Gamma_{\lambda}=\gamma_{\lambda} F_{1}\left((k-q)^{2}\right)-\sigma_{\lambda \rho} \frac{(k-q)_{\rho}}{2 m_{2}} F_{2}\left((k-q)^{2}\right)$,

where $F_{1}\left(k^{2}\right)$ and $F_{2}\left(k^{2}\right)$ are the Dirac and Pauli electromagnetic form factors of the proton. When calculating the corrections to the structure of the nucleus without recoil (to the finite size of the nucleus) we can neglect in the proton propagator its kinetic energy:

$$
\frac{1}{\left(k-p_{2}\right)^{2}-m_{2}^{2}} \approx \frac{1}{-2 m_{2} k^{0}+i 0}, \quad \frac{1}{\left(k+p_{2}-q-p\right)^{2}-m_{2}^{2}} \approx \frac{1}{2 m_{2} k^{0}+i 0} .
$$

In sum, these two expressions give the Dirac delta function $\delta\left(k^{0}\right)$, which allows us to perform an analytic integration with respect to $k^{0}$. Using the polarization vector $\varepsilon_{\tau}(L)$ of the orbital motion from (44) and the muon Dirac bispinor $u(0)$ in the rest frame, we obtain for the $2 P_{1 / 2}$ muon state at the first projection step the following expressions for numerators of direct and crossed amplitudes:

$$
\begin{gathered}
N_{d i r}=\frac{1}{3}\left[\bar{\psi}(0)\left(\gamma_{\omega}-v_{\omega}\right) \gamma_{5}\left(\hat{q}_{1}+m_{1}\right) \gamma_{\mu}\left(\hat{p}_{1}+\hat{k}+m_{1}\right) \gamma_{\nu}\left(\hat{p}_{1}+m_{1}\right) \gamma_{5}\left(\gamma_{\tau}-v_{\tau}\right) \psi(0) \times\right. \\
\left.\bar{v}(0)\left(\hat{p}_{2}-m_{2}\right) \Gamma_{\sigma}\left(\hat{k}-\hat{p}_{2}+m_{2}\right) \Gamma_{\lambda}\left(\hat{q}_{2}-m_{2}\right) v(0)\right], \\
N_{c r}=\frac{1}{3}\left[\bar{\psi}(0)\left(\gamma_{\omega}-v_{\omega}\right) \gamma_{5}\left(\hat{q}_{1}+m_{1}\right) \gamma_{\mu}\left(\hat{p}_{1}+\hat{k}+m_{1}\right) \gamma_{\nu}\left(\hat{p}_{1}+m_{1}\right) \gamma_{5}\left(\gamma_{\tau}-v_{\tau}\right) \psi(0) \times\right. \\
\left.\bar{v}(0)\left(\hat{p}_{2}-m_{2}\right) \Gamma_{\lambda}\left(\hat{k}-\hat{p}_{2}+m_{2}\right) \Gamma_{\sigma}\left(\hat{q}_{2}-m_{2}\right) v(0)\right],
\end{gathered}
$$

where the spinor $\psi(0)$ describes the muon in the rest frame with total momentum $j=1 / 2$. Using the wave functions of the muon $\psi(0)$ and the proton $v(0)$, we introduce the projection operators on states with total angular momentum $F=1$ and $F=0$ in the second step. As a result, we obtain the following expressions in the numerator of the direct two-photon amplitude:

$$
\begin{aligned}
N_{d i r}^{(1)}= & \frac{1}{24} \operatorname{Tr}\left[\gamma_{5}\left(1+\gamma_{0}\right)\left(\gamma_{\omega}-v_{\omega}\right) \gamma_{5}\left(\hat{q}_{1}+m_{1}\right) \gamma_{\mu}\left(\hat{p}_{1}+\hat{k}+m_{1}\right) \gamma_{\nu}\left(\hat{p}_{1}+m_{1}\right)\right. \\
& \left.\gamma_{5}\left(\gamma_{\tau}-v_{\tau}\right)\left(1+\gamma_{0}\right) \gamma_{5}\left(\hat{p}_{2}-m_{2}\right) \Gamma_{\sigma}\left(\hat{k}-\hat{p}_{2}+m_{2}\right) \Gamma_{\lambda}\left(\hat{q}_{2}-m_{2}\right)\right] \\
N_{d i r}^{(2)}= & \frac{1}{72} \operatorname{Tr}\left[\hat{\varepsilon}^{*}\left(1+\gamma_{0}\right)\left(\gamma_{\omega}-v_{\omega}\right) \gamma_{5}\left(\hat{q}_{1}+m_{1}\right) \gamma_{\mu}\left(\hat{p}_{1}+\hat{k}+m_{1}\right) \gamma_{\nu}\left(\hat{p}_{1}+m_{1}\right)\right. \\
& \left.\gamma_{5}\left(\gamma_{\tau}-v_{\tau}\right)\left(1+\gamma_{0}\right) \hat{\varepsilon}\left(\hat{p}_{2}-m_{2}\right) \Gamma_{\sigma}\left(\hat{k}-\hat{p}_{2}+m_{2}\right) \Gamma_{\lambda}\left(\hat{q}_{2}-m_{2}\right)\right],
\end{aligned}
$$

where the new polarization vector $\varepsilon_{\mu}$ in (13) corresponds to the state with $F=1$. Similar expressions for (12)-(13) are obtained for crossed two-photon diagram. It should be emphasized that the advantage of this approach is that further transformations are performed within the Form [37] package, which greatly simplifies the construction of the contribution to the particle interaction operator. As a result, the amplitude correction from (6) -(7) in the energy spectrum of $2^{1} P_{1 / 2}, 2^{3} P_{1 / 2}$ states can be represented in the following integral form:

$$
\Delta E\left(2^{3} P_{1 / 2}\right)=\frac{(Z \alpha)^{2}}{96 \pi^{5} m_{2}} \int \frac{d \mathbf{p}}{(\mathbf{k}-\mathbf{p})^{\mathbf{2}}} R_{21}(p) \int \frac{d \mathbf{q}}{(\mathbf{k}-\mathbf{q})^{\mathbf{2}}} R_{21}(q) \int \frac{d \mathbf{k}}{\mathbf{k}^{2}}\left\{F_{1}\left((\mathbf{k}-\mathbf{p})^{2}\right) F_{2}(\mathbf{k}-\mathbf{q})^{2}\right)
$$




$$
\begin{aligned}
& \times\left[\frac{4}{3 p q}(\mathbf{k p})(\mathbf{k q})-\frac{4 q}{3 p}(\mathbf{k p})-\frac{4 p}{3 q}(\mathbf{k q})+\frac{2}{3}(\mathbf{p q})\left(\frac{p}{q}+\frac{q}{p}\right)\right]+F_{1}\left((\mathbf{k}-\mathbf{p})^{2}\right) F_{1}\left((\mathbf{k}-\mathbf{q})^{2}\right)\left[2 \mathbf{k}^{2} \frac{(\mathbf{p q})}{p q}+\right. \\
& \left.\quad \frac{4}{3 p q}(\mathbf{k p})(\mathbf{k q})+\frac{2 q}{3 p}(\mathbf{k p})\left(1+\frac{3 m_{2}}{2 m_{1}}\right)+\frac{2 p}{3 q}(\mathbf{k q})\left(1+\frac{3 m_{2}}{2 m_{1}}\right)+\frac{5}{3}(\mathbf{p q})\left(\frac{p}{q}+\frac{q}{p}\right)+4 m_{1} m_{2} \frac{(\mathbf{p q})}{p q}\right] \\
& \Delta E\left(2^{1} P_{1 / 2}\right)=\frac{(Z \alpha)^{2}}{32 \pi^{5} m_{2}} \int \frac{d \mathbf{p}}{(\mathbf{k}-\mathbf{p})^{\mathbf{2}}} R_{21}(p) \int \frac{d \mathbf{q}}{(\mathbf{k}-\mathbf{q})^{\mathbf{2}}} R_{21}(q) \int \frac{d \mathbf{k}}{\mathbf{k}^{2}}\left\{F_{1}\left((\mathbf{k}-\mathbf{p})^{2}\right) F_{2}(\mathbf{k}-\mathbf{q})^{2}\right) \\
& \times\left[\frac{4}{p q}(\mathbf{k p})(\mathbf{k q})-\frac{4 q}{p}(\mathbf{k p})-\frac{4 p}{q}(\mathbf{k q})+2(\mathbf{p q})\left(\frac{p}{q}+\frac{q}{p}\right)\right]+F_{1}\left((\mathbf{k}-\mathbf{p})^{2}\right) F_{1}\left((\mathbf{k}-\mathbf{q})^{2}\right)\left[-2 \mathbf{k}^{2} \frac{(\mathbf{p q})}{p q}+\right. \\
& \left.\frac{4}{p q}(\mathbf{k p})(\mathbf{k q})-\frac{q}{p}(\mathbf{k p})\left(6+\frac{m_{2}}{m_{1}}\right)+\frac{p}{q}(\mathbf{k q})\left(6+\frac{m_{2}}{m_{1}}\right)+(\mathbf{p q})\left(\frac{p}{q}+\frac{q}{p}\right)-4 m_{1} m_{2} \frac{(\mathbf{p q})}{p q}\right] .
\end{aligned}
$$

In this work, we set the goal of investigating contributions to the finite size of the nucleus in the fine and hyperfine structure of the P-levels coming from two-photon exchanges. To this end, we use an expansion of form factors for small momenta of the form:

$$
F_{i}\left(\mathbf{k}^{2}\right)=F_{i}(0)+\left.\frac{d F_{i}\left(\mathbf{k}^{2}\right)}{d \mathbf{k}^{2}}\right|_{\mathbf{k}^{2}=\mathbf{0}} \mathbf{k}^{2}+\left.\frac{1}{2} \frac{d^{2} F_{i}\left(\mathbf{k}^{2}\right)}{\left(d \mathbf{k}^{2}\right)^{2}}\right|_{\mathbf{k}^{2}=\mathbf{0}} \mathbf{k}^{4}+\ldots
$$

In what follows, from this expansion we take into account only the terms $\sim r_{E}^{2}$, omitting the terms $\sim r_{E}^{4}$, and so on. After the expansion, our momentum integrals in (14), (15) remain finite and there is no need to introduce any cutoff for them and divide the region of integration into a region of small and large momenta (In contrast at the calculation of the Zemach correction, such expansion leads to a divergent integral and it is necessary to divide the integration region into two parts of large and small momenta). In the region of large momenta, the contribution of the integral is zero because of the angular integration. In the exact calculation of the resulting integrals (without expansion), the contribution of the region of large momenta is suppressed by additional factor $m_{1}^{2} / \Lambda^{2}$ and is of no interest for this study.

Expressions (14)-(15) are the result of the calculation of the amplitudes in the Form package with necessary accuracy. To extract the contribution to the structure of the nucleus in the analytic form, we expand the form factors $F_{1,2}$ in a series, preserving the term proportional to the charge radius squared $r_{E}^{2}$. We take into account that the integral functions in (14) -(15) are symmetric with respect to the replacement $\mathbf{p} \leftrightarrow \mathbf{q}$ and represent the product of form factors as follows:

$$
F_{1}\left((\mathbf{k}-\mathbf{p})^{2}\right) F_{1}\left((\mathbf{k}-\mathbf{q})^{2}\right) \approx 1+\frac{1}{3} r_{E}^{2}(\mathbf{k}-\mathbf{p})^{2}, F_{1}\left((\mathbf{k}-\mathbf{p})^{2}\right) F_{2}\left((\mathbf{k}-\mathbf{q})^{2}\right) \approx \kappa+\frac{\kappa}{3} r_{E}^{2}(\mathbf{k}-\mathbf{p})^{2}
$$

where $\kappa=F_{2}(0)$ is the proton anomalous magnetic moment, $r_{E}^{2}=-\left.6 \frac{d F_{1}\left(k^{2}\right)}{d k^{2}}\right|_{k^{2}=0}=$ $-\left.6 \frac{d F_{2}\left(k^{2}\right)}{d k^{2}}\right|_{k^{2}=0} / \kappa$. Then the result of integration in (14)-(15) is defined by the following integral:

$$
I=\int \frac{d \mathbf{k}}{\mathbf{k}^{2}} \int p d \mathbf{p} R_{21}(p) \int \frac{d \mathbf{q}}{(\mathbf{q}-\mathbf{k})^{2}} \frac{(\mathbf{k q})}{q} R_{21}(q)=4 \pi^{5} W^{4}, \quad W=\mu Z \alpha,
$$


where $\mu=m_{1} m_{2} /\left(m_{1}+m_{2}\right)$. Taking into account (18), we obtain from (14)-(15) analytical expressions for the energy corrections:

$$
\begin{gathered}
\Delta E\left(2^{3} P_{1 / 2}\right)=E_{F}(2 S) \frac{1}{24}(Z \alpha)^{2} m_{1} \mu r_{E}^{2}\left(-\frac{4 \kappa}{1+\kappa}+\frac{3 \frac{m_{2}}{m_{1}}+2}{1+\kappa}\right), \\
\Delta E\left(2^{1} P_{1 / 2}\right)=E_{F}(2 S) \frac{1}{8}(Z \alpha)^{2} m_{1} \mu r_{E}^{2}\left(-\frac{4 \kappa}{1+\kappa}-\frac{\frac{m_{2}}{m_{1}}+6}{1+\kappa}\right),
\end{gathered}
$$

for convenience of presenting the result the Fermi energy for the 2S-level is introduced: $E_{F}(2 S)=\frac{1}{3} \frac{(1+\kappa) \mu^{3} \alpha^{4}}{m_{1} m_{2}}$. Another scheme for adding moments involves first adding the particle spins to the total spin $S$, and then adding $S$ to the orbital angular momentum $L=1$. In this case, the transformation of the wave function of an atom has the form:

$$
\Psi_{J F F_{z}}=\sum_{S}(-1)^{F+S_{1}+L+S_{2}} \sqrt{2 J+1} \sqrt{2 S+1}\left\{\begin{array}{ccc}
S_{2} & S_{1} & S \\
L & F & J
\end{array}\right\} \Psi_{S F F_{z}} .
$$

The connection between the wave functions in a state with total angular momentum $F=1$ in two schemes for adding moments is determined as follows:

$$
\begin{aligned}
& \Psi_{\frac{1}{2} 1 F_{z}}=-\frac{1}{\sqrt{3}} \Psi_{01 F_{z}}+\sqrt{\frac{2}{3}} \Psi_{11 F_{z}}, \\
& \Psi_{\frac{3}{2} 1 F_{z}}=\sqrt{\frac{2}{3}} \Psi_{01 F_{z}}+\frac{1}{\sqrt{3}} \Psi_{11 F_{z}} .
\end{aligned}
$$

The contribution of states $\Psi_{01 F_{z}}, \Psi_{11 F_{z}}$ to the energy spectrum of P-levels has a form:

$$
\begin{gathered}
\Delta E(2 P, S=0, F=1)=\frac{3(Z \alpha)^{2}}{32 \pi^{5} m_{2}} \int \frac{d \mathbf{p}}{(\mathbf{k}-\mathbf{p})^{2}} R_{21}(p) \int \frac{d \mathbf{q}}{(\mathbf{k}-\mathbf{q})^{2}} R_{21}(q) \int \frac{d \mathbf{k}}{\mathbf{k}^{2}} \times \\
\left\{F_{1}\left((\mathbf{k}-\mathbf{p})^{2}\right) F_{2}(\mathbf{k}-\mathbf{q})^{2}\right)\left[\frac{4}{3 p q}(\mathbf{p q}) \mathbf{k}^{2}-\frac{4}{3 p q}(\mathbf{k p})(\mathbf{p q})-\frac{4}{3 p q}(\mathbf{k q})(\mathbf{q p})+\frac{2}{3}(\mathbf{p q})\left(\frac{p}{q}+\frac{q}{p}\right)\right]+ \\
F_{1}\left((\mathbf{k}-\mathbf{p})^{2}\right) F_{1}\left((\mathbf{k}-\mathbf{q})^{2}\right)\left[2 \mathbf{k}^{2} \frac{(\mathbf{p q})}{p q}+\frac{1}{3 p q}(\mathbf{k p})(\mathbf{p q})+\frac{1}{3 p q}(\mathbf{k q})(\mathbf{p q})\left(\frac{m_{2}}{m_{1}}-2\right)+\right. \\
\left.\left.\frac{1}{3 p q}(\mathbf{k q})(\mathbf{p q})\left(\frac{m_{2}}{m_{1}}-2\right)+(\mathbf{p q})\left(\frac{p}{q}+\frac{q}{p}\right)+4 m_{1} m_{2} \frac{(\mathbf{p q})}{3 p q}\right]\right\}= \\
=E_{F}(2 S) \frac{1}{24} m_{1} \mu r_{E}^{2}(Z \alpha)^{2}\left(-\frac{4 \kappa}{1+\kappa}+\frac{\frac{m_{2}}{m_{1}}-2}{1+\kappa}\right) . \\
\Delta E(2 P, S=1, F=1)=-\frac{3(Z \alpha)^{2}}{32 \pi^{5} m_{2}} \int \frac{d \mathbf{p}}{(\mathbf{k}-\mathbf{p})^{2}} R_{21}(p) \int \frac{d \mathbf{q}}{(\mathbf{k}-\mathbf{q})^{2}} R_{21}(q) \int \frac{d \mathbf{k}}{\mathbf{k}^{2}} \times \\
\left\{F_{1}\left((\mathbf{k}-\mathbf{p})^{2}\right) F_{2}(\mathbf{k}-\mathbf{q})^{2}\right)\left[\frac{2}{3 p q}(\mathbf{p q}) \mathbf{k}^{2}-\frac{2}{3 p q}(\mathbf{k p})(\mathbf{k q})\right]+ \\
F_{1}\left((\mathbf{k}-\mathbf{p})^{2}\right) F_{1}\left((\mathbf{k}-\mathbf{q})^{2}\right)\left[-\frac{2}{3 p q}(\mathbf{k p})(\mathbf{p q})-\frac{1}{6 p q}(\mathbf{k p})(\mathbf{p q})\left(\frac{m_{2}}{m_{1}}+2\right)-\right.
\end{gathered}
$$




$$
\begin{gathered}
\left.\left.\frac{1}{6 p q}(\mathbf{k q})(\mathbf{p q})\left(\frac{m_{2}}{m_{1}}+2\right)-\frac{q}{6 p}(\mathbf{k p})\left(\frac{m_{2}}{m_{1}}+2\right)-\frac{p}{6 q}(\mathbf{k q})\left(\frac{m_{2}}{m_{1}}+2\right)-\frac{1}{3}(\mathbf{p q})\left(\frac{q}{p}+\frac{p}{q}\right)-4 m_{1} m_{2} \frac{(\mathbf{p q})}{3 p q}\right]\right\}= \\
=E_{F}(2 S) \frac{1}{12} m_{1} \mu r_{E}^{2}(Z \alpha)^{2} \frac{\frac{m_{2}}{m_{1}}+2}{1+\kappa}
\end{gathered}
$$

where when we add the spin and orbital angular momentum to the total moment $F=1$, the following tensor expression is used $\left(\epsilon^{\beta}\right.$ is the polarization vector of the state with $\left.F=1\right)$ :

$$
\varepsilon_{\rho \sigma}=\frac{i}{\sqrt{2}} \varepsilon_{\rho \sigma \alpha \beta} v^{\alpha} \epsilon^{\beta}
$$

Using further (22)-(23), we can obtain total contribution $\Delta E\left(2^{3} P_{1 / 2}\right), \Delta E\left(2^{3} P_{3 / 2}\right)$ from (24) $-(25)$. It is also necessary to take into account nondiagonal matrix element of the form

$$
\begin{gathered}
\Delta E(S=0, S=1)=<\Psi_{01 F_{z}}\left|\Delta V_{2 \gamma}\right| \Psi_{11 F_{z}}>= \\
\frac{3(Z \alpha)^{2}}{32 \sqrt{2} \pi^{5} m_{2}} \int \frac{d \mathbf{p}}{(\mathbf{k}-\mathbf{p})^{\mathbf{2}}} R_{21}(p) \int \frac{d \mathbf{q}}{(\mathbf{k}-\mathbf{q})^{\mathbf{2}}} R_{21}(q) \int \frac{d \mathbf{k}}{\mathbf{k}^{2}}\left\{F_{1}\left((\mathbf{k}-\mathbf{p})^{2}\right) F_{2}(\mathbf{k}-\mathbf{q})^{2}\right) \times \\
{\left[-\frac{2}{3 p q}(\mathbf{k p})(\mathbf{p q})+\frac{2 q}{3 p}(\mathbf{k p})+\frac{2 p}{3 q}(\mathbf{k q})-\frac{2}{3 p q}(\mathbf{k q})(\mathbf{p q})\right]+} \\
F_{1}\left((\mathbf{k}-\mathbf{p})^{2}\right) F_{1}\left((\mathbf{k}-\mathbf{q})^{2}\right)\left[\frac{1}{3 p q} \frac{m_{2}}{m_{1}}(\mathbf{k p})(\mathbf{p q})+\frac{1}{3 p q} \frac{m_{2}}{m_{1}}(\mathbf{k q})(\mathbf{p q})-\frac{q}{3 p}(\mathbf{k p}) \frac{m_{2}}{m_{1}}-\frac{p}{3 q}(\mathbf{k q}) \frac{m_{2}}{m_{1}}\right]= \\
=E_{F}(2 S) \frac{\sqrt{2}}{24} m_{1} \mu(Z \alpha)^{2} r_{E}^{2}\left(\frac{2 \kappa}{1+\kappa}-\frac{\frac{m_{2}}{m_{1}}}{1+\kappa}\right) .
\end{gathered}
$$

The contribution $\Delta E\left(2^{3} P_{1 / 2}\right)$ has exactly the same form (19), as with another method of adding moments, and the contribution of this interaction to the level $2^{3} P_{3 / 2}$ shift is equal to

$$
\Delta E\left(2^{3} P_{3 / 2}\right)=0
$$

In the case of the state $2^{5} P_{3 / 2}$ with $F=2$, it is convenient to first add the spin moments of the particles, obtaining a state with spin $S=1$, which is described by the polarization vector $\varepsilon_{\mu}(S)$. Then, from the two wave functions $\varepsilon_{\mu}(S)$ and $\varepsilon_{\omega}(L)$, we construct the tensor $\varepsilon_{\mu \omega}$, describing the state with total angular momentum $F=2$. The further summation over the projections of total angular momentum is given by:

$$
\sum_{\gamma} \varepsilon_{\mu \nu}^{*}{ }^{(\gamma)} \varepsilon_{\lambda \omega}^{(\gamma)}=\frac{1}{2}\left(\mathcal{P}_{\mu \lambda} \mathcal{P}_{\nu \omega}+\mathcal{P}_{\mu \omega} \mathcal{P}_{\nu \lambda}\right)-\frac{1}{3} \mathcal{P}_{\mu \nu} \mathcal{P}_{\lambda \omega}
$$

As a result the nuclear structure correction for the state $2^{5} P_{3 / 2}$ has the following integral representation:

$$
\begin{gathered}
\Delta E\left(2^{5} P_{3 / 2}\right)=\frac{3 \alpha^{2}}{32 \pi^{5} m_{2}} \int \frac{R_{21}(p) d \mathbf{p}}{(\mathbf{p}-\mathbf{k})^{2}} \int \frac{R_{21}(q) d \mathbf{q}}{(\mathbf{q}-\mathbf{k})^{2}} \int \frac{d \mathbf{k}}{\mathbf{k}^{2}}\left\{F_{1}\left((\mathbf{k}-\mathbf{p})^{2}\right) F_{1}\left((\mathbf{k}-\mathbf{q})^{2}\right) \times\right. \\
{\left[-\frac{4}{3} \mathbf{k}^{2} \frac{\mathbf{p q}}{p q}+\frac{2}{3} \frac{(\mathbf{k p})(\mathbf{k q})}{p q}-\frac{5}{2} \frac{(\mathbf{k p})(\mathbf{p q})}{p q} \frac{m_{2}}{m_{1}}-9 \frac{(\mathbf{k p})(\mathbf{p q})}{p q}+\mathbf{k p} \frac{q}{p}\left(\frac{5}{6} \frac{m_{2}}{m_{1}}+3\right)-\right.}
\end{gathered}
$$




$$
\begin{gathered}
\left.\frac{(\mathbf{k q})(\mathbf{p q})}{p q}\left(\frac{5}{2} \frac{m_{2}}{m_{1}}+9\right)+\mathbf{k q} \frac{p}{q}\left(\frac{5}{6} \frac{m_{2}}{m_{1}}+3\right)-\frac{1}{3}(\mathbf{p q})\left(\frac{q}{p}+\frac{p}{q}\right)-\frac{20}{3} m_{1} m_{2} \frac{(\mathbf{p q})}{p q}\right] \\
F_{1}\left((\mathbf{k}-\mathbf{p})^{2}\right) F_{2}\left((\mathbf{k}-\mathbf{q})^{2}\right)\left[2 \mathbf{k}^{2} \frac{\mathbf{p q}}{p q}+\frac{2}{3} \frac{(\mathbf{k q})(\mathbf{k p})}{p q}-4 \frac{(\mathbf{k p})(\mathbf{p q})}{p q}-4 \frac{(\mathbf{k q})(\mathbf{p q})}{p q}+\right. \\
\left.\left.\frac{4}{3} \mathbf{k p} \frac{q}{p}+\frac{4}{3} \mathbf{k q} \frac{p}{q}+\frac{4}{3} \mathbf{p q}\left(\frac{p}{q}+\frac{q}{p}\right)\right]\right\} .
\end{gathered}
$$

Using further the expansion of (17) and extracting the correction to the nuclear structure $\left(\sim r_{E}^{2}\right)$, we obtain a cancellation of all terms in (30).

The obtained expressions allow us to present a correction for the nuclear structure separately in the fine and hyperfine structure of the P-energy levels in the form:

$$
\begin{gathered}
\Delta E\left(2 P_{1 / 2}\right)=\Delta E\left(2 P_{1 / 2}(F=0)\right)+\frac{3}{4} \Delta E^{h f_{s}}\left(2 P_{1 / 2}\right), \\
\Delta E\left(2 P_{3 / 2}\right)=\Delta E\left(2 P_{3 / 2}(F=1)\right)+\frac{5}{8} \Delta E^{h f s}\left(2 P_{3 / 2}\right), \Delta E_{s t r}^{f s}\left(2 P_{3 / 2}-2 P_{1 / 2}\right)=-0.0014 \mu \mathrm{eV}, \\
\Delta E_{s t r}^{h f s}\left(2 P_{1 / 2}\right)=0.2893 \mu \mathrm{eV}, \quad \Delta E_{s t r}^{h f_{s}}\left(2 P_{3 / 2}\right)=0 \mu \mathrm{eV} .
\end{gathered}
$$

It is useful to compare our results with the available calculations of corrections to the finite size of the nucleus for P-levels in [19, 38, 39]. For $\mathrm{n}=2$, using [19, 38, 39], we get $\Delta E\left(2 P_{1 / 2}\right)=(Z \alpha)^{6} r_{E}^{2} \mu^{3} / 64$. From our formulas (19), (20), (24), (25), (27), (31) it follows that in the leading order in $m_{1} / m_{2}$ the analogous contribution is equal to $\Delta E\left(2 P_{1 / 2}\right)=$ $(Z \alpha)^{6} r_{E}^{2} \mu^{3} / 48$ and differs from the previous one by the factor $4 / 3$. Nuclear size correction of order $m(Z \alpha)^{6} r_{E}^{2}$ was obtained in [19, 38, 39] in $1 \gamma$-interaction. In this paper we have constructed the potentials of two-photon exchange, which effectively takes into account the contribution of the one-photon interaction, since the iterative term that we take into account is related with a point proton. Thus, the calculations of two-photon exchange amplitudes that have been carried out refine the previous result for P-levels of order $m(Z \alpha)^{6} r_{E}^{2}$.

When obtaining the numerical results in Table I. we use the value of the proton charge radius $r_{E}=0.84087 \mathrm{fm}$. We note that the results (18), (19), (24) themselves are obtained in an analytical form, where the factor $r_{E}^{2}$ is extracted. Therefore, when comparing the theory and experiment and obtaining a more accurate value of $r_{E}$, these expressions can be used, forming a common coefficient before $r_{E}^{2}$ from all possible corrections. Numerical values of the contributions (29)-(32) are presented in Table [. They refine the position of energy levels $2^{1} P_{1 / 2}, 2^{3} P_{1 / 2}, 2^{3} P_{3 / 2}, 2^{5} P_{3 / 2}$ and may be needed for more exact comparison of theory and experiment. There is no contribution $\sim r_{E}^{2}$ to the energy of $2 P_{3 / 2}$ state. There is another type of two-photon interactions that contribute to the structure of P-states. It is considered in the next section.

\section{CONTRIBUTION OF AXIAL VECTOR MESONS TO MUON-PROTON INTERACTION}

Corrections in the fine and hyperfine structure of P-levels also arise as a result of twophoton interaction of another type, shown in Fig. 2. In this case, the effect of two-photon interaction leads to the appearance of a meson exchange between the proton and the muon. The contribution of pseudoscalar mesons in the case of S-, P-states was calculated in [16, 
40 42]. The contribution of axial vector mesons to the hyperfine structure of the S-state spectrum, which was calculated in [17], is significant. Therefore, it is important to consider this contribution also to the $2 P_{1 / 2}, 2 P_{3 / 2}$ level shifts.

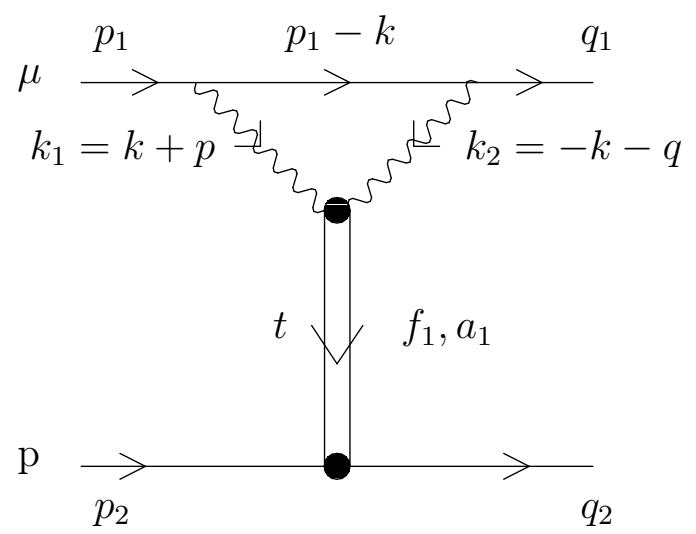

FIG. 2: Contribution of axial vector meson to the amplitude of muon-proton interaction.

An important role in the interaction amplitude in Fig. 2 is played by the vertex of the transition of two photons into an axial vector meson. For it, the following parameterization is valid [43, 44]:

$$
T^{\mu \nu \alpha}=4 \pi i \alpha \varepsilon_{\mu \nu \alpha \beta}\left(k_{1}^{\beta} k_{2}^{2}-k_{2}^{\beta} k_{1}^{2}\right) F_{A V \gamma^{*} \gamma^{*}}^{(0)}\left(t^{2}, k_{1}^{2}, k_{2}^{2}\right),
$$

where $t=p-q$. If $k_{1}^{2}=0, k_{2}^{2}=0$, then the vertex function (33) vanishes. Introducing the projection operators to the state $2 P_{1 / 2}$ as before in (10)-(11), we represent the interaction amplitude of particles in this state in the form:

$$
\begin{gathered}
\mathcal{M}\left(2^{3} P_{1 / 2}\right)=\frac{\alpha^{2} g_{A V P P}}{512 \pi^{3} m_{1}^{2} m_{2}^{2}} \int d^{4} k \operatorname{Tr}\left\{\hat{\varepsilon}\left(1+\gamma_{0}\right)\left(\gamma_{\lambda}-v_{\lambda}\right) \gamma_{5}\left(\hat{q}_{1}+m_{1}\right) \gamma_{\mu}\left[m_{1} \hat{v}-\hat{k}+m_{1}\right] \gamma_{\nu} \times\right. \\
\left(\hat{p}_{1}+m_{1}\right) \gamma_{5}\left(\gamma_{\omega}-v_{\omega}\right)\left(1+\gamma_{0}\right) \hat{\varepsilon}\left(\hat{p}_{2}-m_{2}\right) \gamma_{\alpha} \gamma_{5}\left(\hat{q}_{2}-m_{2}\right) n_{q}^{\lambda} n_{p}^{\omega} F_{A \gamma^{*} \gamma^{*}}^{(0)}\left(t^{2}, k_{1}^{2}, k_{2}^{2}\right) \times \\
\left.\frac{\varepsilon_{\mu \nu \alpha \beta}}{k^{2}-2 m_{1} k_{0}}\left[\frac{(k+p)_{\beta}}{(k+p)^{2}}+\frac{(k+q)_{\beta}}{(k+q)^{2}}\right] \frac{1}{\mathbf{t}^{2}+M_{A}^{2}}\right\},
\end{gathered}
$$

where $g_{A V P P}$ is the coupling constant of the axial vector meson and proton. To extract the leading order contribution in $\alpha$ we can make approximately the following replacements: $(k+p)^{\beta} /(k+p)^{2} \rightarrow\left(k^{\beta}+p^{\beta}\right) / k^{2},(k+q)^{\beta} /(k+q)^{2} \rightarrow\left(k^{\beta}+q^{\beta}\right) / k^{2}$. Then the interaction amplitude gives the contribution to the energy shift of $2 P_{1 / 2}$ level, which can be presented in integral form:

$$
\begin{aligned}
\Delta E\left(2^{3} P_{1 / 2}\right)= & \frac{\alpha^{2} g_{A V P P}}{4 \pi^{3}} \int d \mathbf{p} R_{21}(p) \int d \mathbf{q} R_{21}(q) \int \frac{d k}{k^{2}\left(k^{2}-2 m_{1} k^{0}\right)} F_{A V \gamma^{*} \gamma^{*}}^{(0)}\left(0, k^{2}, k^{2}\right) \times \\
& \left\{-\frac{8}{3}(k p)(k q) \frac{1}{p q}+\frac{4}{3} k_{0}^{2}(\mathbf{p q}) \frac{1}{p q}+\frac{2 k_{0}}{m_{1}} p q-\frac{4}{3} p q\right\} \frac{1}{\mathbf{t}^{2}+M_{A}^{2}} .
\end{aligned}
$$

We left terms in brackets of expression (35) that formally have a different order in $\alpha$, taking into account that $p \sim \mu \alpha, q \sim \mu \alpha$. The subsequent angular integration in the momentum 
space shows that their order is equalized and becomes equal to $\alpha^{5}$. All the momentum integrals in (35) can be calculated analytically by using the vector dominance model for the function $F_{A V \gamma^{*} \gamma^{*}}^{(0)}\left(0, k^{2}, k^{2}\right)$ [45-48]:

$$
\begin{aligned}
& F_{A V \gamma^{*} \gamma^{*}}^{(0)}\left(0, k^{2}, k^{2}\right)=\frac{F_{A V \gamma^{*} \gamma^{*}}^{(0)}(0,0)}{\left(1+\frac{k^{2}}{\Lambda^{2}}\right)^{2}}=F_{A V \gamma^{*} \gamma^{*}}^{(0)}(0,0) F\left(k^{2}\right), \\
& I_{1}=\int \frac{d k\left(k^{2}+2 k_{0}^{2}\right)}{\left(k^{4}+4 m_{1}^{2} k_{0}^{2}\right)} F^{2}\left(k^{2}\right)=-\Lambda^{2} \frac{\pi^{2}}{2}\left[1+\left(\frac{5}{2}-2 \ln 2+2 \ln \frac{2 m_{1}}{\Lambda}\right) \frac{4 m_{1}^{2}}{\Lambda^{2}}\right], \\
& I_{2}=\int \frac{d k\left(3 k_{0}^{2}-k^{2}\right)}{k^{2}\left(k^{4}+4 m_{1}^{2} k_{0}^{2}\right)} F^{2}\left(k^{2}\right)=4 \pi^{2}\left[-\frac{11}{48}-\frac{1}{8} \ln 2+\frac{1}{8} \ln \frac{2 m_{1}}{\Lambda}+\right. \\
& \left.\frac{1}{96}\left(-31+24 \ln 2-24 \ln \frac{2 m_{1}}{\Lambda}\right) \frac{4 m_{1}^{2}}{\Lambda^{2}}\right], \\
& J_{1}=\int d \mathbf{p} R_{21}(p) \int d \mathbf{q} R_{21}(q) \frac{\mathbf{p q}}{p q} \frac{1}{(\mathbf{p}-\mathbf{q})^{2}+M_{A}^{2}}=\frac{2 \pi^{3} W^{5}}{\left(M_{A}+W\right)^{4}}, \quad W=\mu Z \alpha, \\
& J_{2}=\int p d \mathbf{p} R_{21}(p) \int q d \mathbf{q} R_{21}(q) \frac{1}{(\mathbf{p}-\mathbf{q})^{2}+M_{A}^{2}}=\frac{\pi^{3} W^{5} M_{A}^{2}}{\left(M_{A}+W\right)^{4}}\left[3+\frac{4 W}{M_{A}}+\frac{3 W^{2}}{2 M_{A}^{2}}\right],
\end{aligned}
$$

where the values of parameter $\Lambda$ for three axial vector mesons $f_{1}(1285), a_{1}(1260), f_{1}(1420)$ are equal $1.040 \mathrm{GeV}, 1.040 \mathrm{GeV}$ and $0.926 \mathrm{GeV}$ correspondingly (see also our Table I from [17]). Here and below, we indicate the expansion of the exact result in powers of the parameter $m_{1} / \Lambda$ up to terms of the second order. An important feature of the integral (39) is that it is zero if $(\mathbf{p}-\mathbf{q})^{2}$ is neglected in the denominator (39). This leads to an increase in the order of the contribution to $\alpha^{5}$. The total analytical result and numerical value of the contribution (35) are equal to

$$
\begin{gathered}
\Delta E\left(2^{3} P_{1 / 2}\right)=\frac{\mu^{5} \alpha^{7} g_{A V P P} F^{(0)}(0,0) \pi^{2}}{M_{A}^{2}}\left\{-\frac{\Lambda^{2}}{M_{A}^{2}}\left[\frac{1}{6}+\frac{2 m_{1}^{2}}{3 \Lambda^{2}}\left(\frac{5}{2}+2 \ln \frac{m_{1}}{\Lambda}\right)\right]-\right. \\
\left.-\frac{11}{12}+\frac{1}{2} \ln \frac{m_{1}}{\Lambda}+\frac{1}{6}\left(-31-24 \ln \frac{m_{1}}{\Lambda}\right) \frac{m_{1}^{2}}{\Lambda^{2}}\right\}=-0.0001 \mu \mathrm{eV} .
\end{gathered}
$$

Numerical value of the contribution (41) is obtained in the case of $f_{1}(1285)$ meson (see the values of corresponding parameters in [17]). It is very small in comparison with the analogous contribution to the shifts of the S-levels, since the order of the contribution increases due to the additional factor $\alpha^{2}$. The contribution of another axial vector meson $a_{1}(1260)$ has approximately the same value, and the total contribution of all AV-mesons is shown in Table I. Similar results for the interaction amplitude and energy shift in the $2^{1} P_{1 / 2}$ state have the form:

$$
\begin{gathered}
\mathcal{M}\left(2^{1} P_{1 / 2}\right)=\frac{\alpha^{2} g_{A V P P}}{4 \pi^{3}} \int \frac{d k F_{A \gamma^{*} \gamma^{*}}^{(0)}\left(k^{2}, k^{2}\right)}{k^{2}\left(k^{2}-2 m_{1} k_{0}\right)}\left[-8 \frac{(k p)(k q)}{p q}+4 k_{0}^{2} \frac{\mathbf{p q}}{p q}+2 p q\left(3 \frac{k_{0}}{m_{1}}-2\right)\right] \frac{1}{\mathbf{t}^{2}+M_{A}^{2}}, \\
\Delta E\left(2^{1} P_{1 / 2}\right)=-\frac{\mu^{5} \alpha^{7} g_{A V P P} F^{(0)}(0,0) \pi^{2}}{2 M_{A}^{2}}\left\{\frac{\Lambda^{2}}{M_{A}^{2}}\left[1+\frac{4 m_{1}^{2}}{\Lambda^{2}}\left(\frac{5}{2}+\ln \frac{m_{1}}{\Lambda}\right)\right]+\right.
\end{gathered}
$$




$$
\left.+\frac{11}{2}-3 \ln \frac{m_{1}}{\Lambda}+\frac{m_{1}^{2}}{\Lambda^{2}}\left(31+24 \ln \frac{m_{1}}{\Lambda}\right)\right\} .
$$

Numerical value of contribution (43) is presented in Table \.

To calculate the analogous contribution in the case of $2 P_{3 / 2}$ state, we use the expansion (23), in which the projection on states $\Psi_{01 F_{z}}$ and $\Psi_{11 F_{z}}$ is also performed within the tensor method. As a result, the muon and proton interaction amplitudes for these states have the form:

$$
\begin{aligned}
& \mathcal{M}_{01 F_{z}}=\frac{3 \alpha^{2} g_{A V P P}}{4 \pi^{3}} \int \frac{d k F_{A \gamma^{*} \gamma^{*}}^{(0)}\left(k^{2}, k^{2}\right)}{k^{2}\left(k^{2}-2 m_{1} k_{0}\right)}\left[\frac{4}{3} \frac{\mathbf{p q}}{p q}\left(k_{0}^{2}+2 k^{2}\right)+\frac{2}{3} \frac{(\mathbf{p q})^{2}}{p q}\left(\frac{k_{0}}{m_{1}}-2\right)\right] \frac{1}{\mathbf{t}^{2}+M_{A}^{2}}, \\
& \mathcal{M}_{11 F_{z}}=\frac{3 \alpha^{2} g_{A V P P}}{8 \pi^{3}} \int \frac{d k F_{A \gamma^{*} \gamma^{*}}^{(0)}\left(k^{2}, k^{2}\right)}{k^{2}\left(k^{2}-2 m_{1} k_{0}\right)}\left[\frac{2}{3} p q \frac{k_{0}}{m_{1}}-2 k^{2} \frac{\mathbf{p q}}{p q}+\frac{2}{3} \frac{k_{0}}{m_{1}} \frac{(\mathbf{p q})^{2}}{p q}\right] \frac{1}{\mathbf{t}^{2}+M_{A}^{2}} .
\end{aligned}
$$

The subsequent integration over the momenta $k, \mathbf{p}, \mathbf{q}$ in the calculation of the energy contributions can be performed analytically:

$$
\begin{gathered}
I_{3}=\int \frac{d k k_{0}^{2}}{k^{2}\left(k^{4}+4 m_{1}^{2} k_{0}^{2}\right)} F^{2}\left(k^{2}\right)=\frac{\pi^{2}}{12}\left[-7-6 \ln \frac{m_{1}}{\Lambda}+\frac{2 m_{1}^{2}}{\Lambda^{2}}\left(-23-24 \ln \frac{m_{1}}{\Lambda}\right)\right], \\
I_{3}=\int \frac{d k k^{2}}{\left(k^{4}+4 m_{1}^{2} k_{0}^{2}\right)} F^{2}\left(k^{2}\right)=\frac{\pi^{2}}{3} \Lambda^{2}\left[1+\frac{m_{1}^{2}}{\Lambda^{2}}\left(7+6 \ln \frac{m_{1}}{\Lambda}\right)\right] .
\end{gathered}
$$

TABLE I: Numerical values of corrections of two-photon interactions to the energy spectrum of P-states in muonic hydrogen.

\begin{tabular}{|c|c|c|}
\hline State & $\begin{array}{c}\text { Correction of } 2 \gamma \text { exchange } \\
\text { amplitudes, } \mu \mathrm{eV}\end{array}$ & $\begin{array}{c}\text { Correction of axial meson exchange } \\
\text { amplitudes, } \mu \mathrm{eV}\end{array}$ \\
\hline $2^{1} P_{1 / 2}$ & -0.2184 & 0.0005 \\
\hline $2^{3} P_{1 / 2}$ & 0.0709 & -0.0002 \\
\hline $2^{3} P_{3 / 2}$ & 0 & -0.00005 \\
\hline $2^{5} P_{3 / 2}$ & 0 & 0.00003 \\
\hline
\end{tabular}

When calculating the average values of axial vector meson exchange amplitudes by means of (23), it is necessary to calculate off-diagonal terms of the form $\left\langle\Psi_{01 F_{z}}|\mathcal{M}| \Psi_{11 F_{z}}\right\rangle$. Denoting such a contribution $\mathcal{M}_{01 F_{z}, 11 F_{z}}$, we obtain the following expression for it:

$\mathcal{M}_{01 F_{z}, 11 F_{z}}=\frac{3 \alpha^{2} g_{A V P P}}{4 \sqrt{2} \pi^{3}} \int \frac{d k F_{A \gamma^{*} \gamma^{*}}^{(0)}\left(k^{2}, k^{2}\right)}{k^{2}\left(k^{2}-2 m_{1} k_{0}\right)}\left[-\frac{2}{3} p q \frac{k_{0}}{m_{1}}+\frac{2}{3} p q+\frac{2}{3}\left(\frac{k_{0}}{m_{1}}-1\right) \frac{(\mathbf{p q})^{2}}{p q}\right] \frac{1}{\mathbf{t}^{2}+M_{A}^{2}}$.

Calculating then the contribution to the energy spectrum from (48) as well as for the diagonal terms, we obtain:

$$
\Delta E_{01 F_{z}, 11 F_{z}}\left(2^{3} P_{3 / 2}\right)=\frac{\mu^{5} \alpha^{7} g_{A V P P} \pi^{2} F_{A \gamma^{*} \gamma^{*}}^{(0)}(0,0)}{3 \sqrt{2} M_{A}^{2}}\left(1-3 \ln \frac{m_{1}}{\Lambda}+\frac{4 m_{1}^{2}}{\Lambda^{2}}\right) .
$$


Total numerical value for the level $2^{3} P_{3 / 2}$, which is determined by (44), (45), (49), is presented in Table I. A similar contribution to the energy level $2^{5} P_{3 / 2}$ shift can be obtained, if in the original amplitude of the muon-proton interaction we perform successive projection onto the state of particles with total spin $S=1$, then the state of total momentum $F=2$. In this case, the muon-proton state is described by the tensor $\varepsilon_{\mu \nu}$, which occurs when two polarization vectors $\varepsilon_{\mu}(L)$ and $\varepsilon_{\nu}(S)$ are added. Summation over the polarizations is carried out with the help of (29). After all the simplifications, the amplitude of particle interaction for $2^{5} P_{3 / 2}$ state is represented in the form:

$$
\begin{gathered}
\mathcal{M}\left(2^{5} P_{3 / 2}\right)=\frac{3 \alpha^{2} g_{A V P P}}{20 \pi^{3}} \int \frac{d k F_{A \gamma^{*} \gamma^{*}}^{(0)}\left(k^{2}, k^{2}\right)}{k^{2}\left(k^{4}-4 m_{1}^{2} k_{0}^{2}\right)}\left[\frac{2}{3} p q\left(2 k^{2}-3 k_{0}^{2}\right)+\frac{2}{3} p q-\right. \\
\left.\frac{1}{3} \frac{(\mathbf{p q})}{p q} k^{2}\left(13 k^{2}+8 k_{0}^{2}\right)+2 \frac{(\mathbf{p q})^{2}}{p q}\left(3 k_{0}^{2}-2 k^{2}\right)\right] \frac{1}{\mathbf{t}^{2}+M_{A}^{2}}
\end{gathered}
$$

and the corresponding contribution to the energy spectrum is included in Table I.

\section{CONCLUSION}

In this paper we study the contribution of some two-photon interactions to the muon and proton interaction potential and into the fine and hyperfine structure of the energy spectrum for P-states. In the framework of the quasipotential method in quantum electrodynamics, using the technique of projection operators on the states of two particles with a definite spin and total angular momentum, we construct the particle interaction operators (14), (15), (34) and obtain analytical expressions for contributions to the fine and hyperfine structure of the P-energy levels. On their basis, numerical estimates of contributions are obtained, which we present in Table I. The method of projection operators allows us to represent the interaction amplitudes of particles in a covariant form and use computer methods for calculating Feynman amplitudes for a further construction of the particle interaction operator in a definite state, which is extremely important in the higher orders of perturbation theory.

A complete picture of the energy levels of muonic hydrogen with $n=2$ was described in [3] in connection with the experiment of the CREMA collaboration. We investigate corrections to the structure of the nucleus of order $\alpha^{6}$ proportional to $r_{E}^{2}$ in the case of P-levels. For the Lamb shift two-photon exchange amplitudes give a correction for the structure of the nucleus, which is expressed in terms of proton electromagnetic form factors (third Zemach). The same two-photon exchange amplitudes give another important contribution, which is known as a correction to the proton polarizability. In the case of the Lamb shift, the numerical values of these contributions to the structure and polarizability of the nucleus are comparable in magnitude. In this connection, it can be expected that in the case of P-states the nuclear reactions of the electroproduction of $\pi-, \eta$-mesons and nucleon resonances can contribute comparable in magnitude to the considered here correction of order $\alpha^{6}$ to the structure of the nucleus.

The contributions of two-photon exchange amplitudes and amplitudes with the exchange of axial vector meson, studied by us, give essentially different numerical values of the Plevel shifts. This is due to the fact that these two-photon interactions lead to corrections of different orders in the energy spectrum: two-photon exchange interactions contribute to a finite nuclear size of the order of $\alpha^{6}$, and the exchange of the axial vector meson produces 
a contribution of order $\alpha^{7}$. In this connection, it can be said that the corrections to the structure of the nucleus from two-photon exchange amplitudes are more significant for a precise comparison with the experimental data because their numerical values are of the order of $0.0001 \mathrm{meV}$ (see Table I). Recall that to explain the puzzle of the proton radius, a contribution of about $0.3 \mathrm{meV}$ is needed. In our calculations of the contribution of axial vector mesons, we take into account the contributions of $f_{1}(1285), a_{1}(1260), f_{1}(1420)$ as in [17]. Their total contribution is presented in Table I. Theoretical uncertainties are not explicitly indicated in Table I. For the correction $\sim r_{E}^{2}$, the error is determined by the error in the value of the charge radius of the proton. If we take two values for the charge radius $r_{E}^{C O D A T A}=0.8775(51) \mathrm{fm}$ and $r_{E}^{C R E M A}=0.84087(39) \mathrm{fm}$, then the difference in the magnitude of this correction is about 9 percent. From the experimental data and model approximations for constructing the transition form factor and the interaction potential of particles for axial vector exchange, we estimate the error in calculating the contribution of axial vector mesons approximately in 50 percent [17].

It should be noted that the axial vector meson exchange mechanism, which contributes to the hyperfine structure of the hydrogen atom spectrum, was discussed many years ago in [49 51]. The most important role for the numerical calculation of the contribution of lightby-light scattering is played by the form factor of the transition of two photons into an axial vector meson. We used for it, just like in the previous paper [17], the dipole parametrization with respect to the 4-momentum of each photon, obtained in the experiments of the collaboration L3 [46 48]. The experimental data of L3 are limited by a rather small kinematic region, so new measurements of transition form factors are extremely necessary. Such experiments can be performed by the BESIII and BELLEII collaborations. The mechanism of interaction of a muon and a nucleus, which is related to effective meson exchange studied in this paper and also in previous publications [16 18], is of great importance for the refinement of the position of the S- and P-energy levels of muonic atoms. Along with the exchange of pseudoscalar and axial vector mesons, it is of interest to consider the exchange of scalar and tensor light mesons, the number of which is significant. Our work in this direction is in progress.

\section{Acknowledgments}

The work is supported by Russian Science Foundation (grant No. RSF 18-12-00128) and Russian Foundation for Basic Research (grant No. 18-32-00023) (F.A.M.).

[1] R. Pohl, A. Antognini, F. Nez et al., Nature 466, 213 (2010).

[2] A. Antognini et al., Science 339, 417 (2013).

[3] A. Antognini et al., Ann. Phys. (NY) 331, 127 (2013).

[4] R. Pohl, F. Nez, L. M. P. Fernandes et al., Science 353, 669 (2016).

[5] Y. Ma et al., Int. J. Mod. Phys. Conf. Ser. 40, 1660046 (2016).

[6] A. Adamczak et al. [FAMU Collaboration], JINST 11, no. 05, P05007 (2016).

[7] R. Pohl [CREMA Collaboration], J. Phys. Soc. Jap. 85, 091003 (2016).

[8] P. J. Mohr, D. B. Newell, and B. N. Taylor (CODATA Recommended Values of the Fundamental Physical Constants: 2014) Rev. Mod. Phys. 88, 035009 (2016). 
[9] I. Sick, Prog. Part. Nucl. Phys. 67, 473 (2012).

[10] C. E. Carlson, Prog. Part. Nucl. Phys. 82, 59 (2015).

[11] A. Beyer et al., Science 358, 79 (2017).

[12] H. Fleurbaey et al. Phys. Rev. Lett. 120, 183001 (2018).

[13] R. N. Faustov, A. P. Martynenko, G. A. Martynenko and V. V. Sorokin, Phys. Lett. B 733, 354 (2014).

[14] R. N. Faustov, A. P. Martynenko, G. A. Martynenko and V. V. Sorokin, Phys. Rev. A 90, 012520 (2014).

[15] R.N. Faustov, A.P. Martynenko, F.A. Martynenko, and V.V. Sorokin, Phys. Lett. B 775, 79 (2017).

[16] A. E. Dorokhov, N. I. Kochelev, A. P. Martynenko, F. A. Martynenko, and R. N. Faustov, Phys. Particles and Nuclei Lett., 14, No.6, 857 (2017); arXiv:1704.07702 [hep-ph].

[17] A. E. Dorokhov, N. I. Kochelev, A. P. Martynenko, F. A. Martynenko, and A. E. Radzhabov, Phys. Lett. B 776, 105 (2018).

[18] A. E. Dorokhov, N. I. Kochelev, A. P. Martynenko, F. A. Martynenko, A. E. Radzhabov, and R. N. Faustov, J. Phys.: Conf. Series 938, 012042 (2017).

[19] M. I. Eides, H. Grotch and V. A. Shelyuto, Theory of Light Hydrogenic Bound States, Springer Tracts in Modern Physics, V. 222 (Springer, Berlin, Heidelbeg, New York, 2007).

[20] H. Grotch and D.R. Yennie, Rev. Mod. Phys. 41, 350 (1969).

[21] E. Borie, Ann. Phys. 327, 733 (2012).

[22] C.E. Carlson and M. Vanderhaeghen, Annu. Rev. Nucl. Part. Sci. 57, 171 (2007).

[23] O. Tomalak and M. Vanderhaeghen, Eur. Phys. J. A 51, 24 (2015).

[24] O. Tomalak and M. Vanderhaeghen, Phys. Rev. D 90, 013006 (2014).

[25] C. Peset and A. Pineda, Nucl. Phys. B 887, 69 (2014).

[26] C. Peset and A. Pineda, JHEP 04 (2017) 060.

[27] A. P. Martynenko and R. N. Faustov, J. Exp. Theor. Phys. 98, 39 (2004).

[28] R. N. Faustov, A. P. Martynenko, G. A. Martynenko, and V.V. Sorokin, Phys. Rev. A 92, $052512(2015)$.

[29] A. A. Krutov, A. P. Martynenko, F. A. Martynenko, and O. S. Sukhorukova, Phys. Rev. A 94, 062505 (2016).

[30] V. B. Berestetskii, E. M. Lifshits and L. P. Pitaevskii, Quantum Electrodynamics, (Nauka, Moscow, 1980).

[31] K. Pachucki, Phys. Rev. A 53, 2092 (1996).

[32] U. D. Jentschura, Ann. Phys. 326, 500 (2011).

[33] S. G. Karshenboim, V. G. Ivanov, E. Yu. Korzinin, and V. A. Shelyuto, Phys. Rev. A 81, 060501 (2010).

[34] S. G. Karshenboim, E. Yu. Korzinin, V. A. Shelyuto, and V. G. Ivanov, J. Phys. Chem. Ref. Data 44, 031202 (2015).

[35] B. Franke et al., Eur. Phys. J. D 71, 341 (2017).

[36] J.J. Krauth et al., Annals Phys. 366, 168 (2016).

[37] J. A. M. Vermaseren, FORM, arXiv:math-ph/0010025.

[38] J.L. Friar, Ann. Phys. 122, 151 (1979).

[39] A.P. Martynenko, A.A. Krutov and R.N. Shamsutdinov, Phys. Atom. Nucl. 77, 786 (2014).

[40] F. Hagelstein and V. Pascalutsa, PoS CD15 077 (2016).

[41] H. Q. Zhou and H. R. Pang, Phys. Rev. A 92, 032512 (2015).

[42] N. T. Huong, E. Kou and B. Moussallam, Phys. Rev. D 93, 114005 (2016). 
[43] R. N. Cahn, Phys. Rev. D 35, 3342 (1987).

[44] V. Pascalutsa, V. Pauk and M. Vanderhaeghen, Phys. Rev. D 85, 116001 (2012).

[45] G. A. Schuler, F. A. Berends and R. van Gulik, Nucl. Phys. B 523, 423 (1998).

[46] P. Achard et al. [L3 Collaboration], Phys. Lett. B 526, 269 (2002).

[47] P. Achard et al. [L3 Collaboration], JHEP 03, 018 (2007)

[48] H. Aihara et al., Phys. Rev. D 38, 1 (1988).

[49] D. F. Flamm and W. Kummer, Nuovo Cim. 28, 1579 (1963).

[50] S. Fenster, R. Koberle and Y. Nambu, Phys. Lett. 19, 513 (1965).

[51] S.D. Drell and J.D. Sullivan, Phys. Lett. 19, 516 (1965). 